Revista Investigaciones Turísticas, no 13, pp. 164-177

ISSN: 2174-5609

DOI. http://dx.doi.org/10.14198/INTURI2017.13.08

†Tr Investigaciones

$\Delta \Rightarrow$ TURISTICAS

\title{
Preferencias climáticas para la práctica del senderismo en España: resultados preliminares
}

\author{
Emilio Martínez Ibarra \\ emibarra@ugr.es \\ Rubén Pardo Martínez \\ rpm007@correo.ugr.es \\ Universidad de Granada
}

\section{RESUMEN}

El presente trabajo tiene por objeto dar a conocer una primera aproximación inédita sobre las preferencias climáticas para la práctica del senderismo en España. Para ello se ha puesto en práctica la técnica de la encuesta, procedimiento de uso habitual en climatología turística. El cuestionario se ha elaborado teniendo en cuenta el marco teórico y metodológico en climatología turística, basado en la influencia de las facetas atmosféricas en el disfrute, confort, salud y riesgo de los turistas. Los resultados reflejan ciertas particularidades en las preferencias climáticas de quiénes practican el senderismo sobre otras actividades turísticas. En futuros trabajos se pretende seguir ahondando en el conocimiento de la relación clima-senderismo.

Palabras clave: climatología turística; senderismo; España; preferencias climáticas 


\section{Climate preferences for hiking in Spain: preliminary results}

\section{ABSTRACT}

This study is the first of its kind in addressing the climatic preferences for hiking in Spain. To do so, the survey technique has been used, which is a procedure that is commonly used in tourism climatology. The design of the questionnaire takes into account the theoretical and methodological framework in tourism climatology, based on the influence that atmospheric facets have on the enjoyment, comfort, health and risk of the tourists. The results reflect certain peculiarities in the climatic preferences of those who practice hiking in relation to other tourist activities. In future research we will continue working on expanding our knowledge of the relationship between climate and hiking.

Key words: tourism climatology; hiking; Spain; climate preferences

\section{INTRODUCCIÓN}

El turismo es una de las actividades económicas más importantes a nivel mundial, que además ha mostrado fortaleza a las distintas crisis económicas internacionales (Martínez-lbarra y Gómez-Martín, 2013; Martínez-lbarra, E., 2016). Así también lo demuestran los datos publicados por la Organización Mundial del Turismo (UNWTO) en 2016. A lo largo del 2015 los flujos de turismo internacional se incrementaron por encima de la media, por sexto año consecutivo. Se registró así un record de 1.184 millones de turistas internacionales. Entre 2014 y 2015 el incremento fue del 4\%, siendo más significativo en determinadas zonas, tal es el caso de Europa. Las expectativas para el 2016 vaticinan también un incremento del 4\%.

De otro lado, el tiempo y el clima juegan un importante papel en el fenómeno turístico (Mintel International Group, 1991; Lohmann y Kaim, 1999; Kozak, 2002; Hamilton y Lau, 2005; Gössling et al., 2006). Bien es cierto que, dicha relación dependerá del tipo de actividad que se practique, por ello se puede establecer la diferenciación entre actividades turísticas sensibles y, por otro, sensibles y dependientes del tiempo y el clima (Smith, 1993; Gómez-Martín, 2005). Un aspecto que conviene trascender es que la relación clima y turismo es bidireccional (el turismo también afecta al clima). $Y$ es que, si bien el clima era considerado como un recurso turístico renovable (Besancenot, 1990), Gómez-Martín ya cuestionaba esta presunción diez años después (ver Gómez-Martín, 2000).

A pesar de los esfuerzos realizados durante las últimas décadas, resumidos en el trabajo de Martínez-Ibarra y Gómez-Martín (2013), aún resulta patente la necesidad de profundizar en el tema que aquí preocupa: la climatología turística. Entre las posibles causas de dicho requerimiento quizás sea necesario traer a colación la opinión de Nicholls y Holecek (2008): estos autores indican que la relación entre clima y turismo se basa, a menudo, en asunciones y anécdotas empíricas, y no en datos objetivos. La necesidad de seguir investigando es aún más 
evidente en turismo activo, tal es el caso de la relación tiempo, clima y senderismo. En este sentido, Perry (2004) realizó una síntesis general sobre el estudio de la relaciones entre el clima y diversas actividades deportivas.

Ciertamente, esta actividad (la práctica del senderismo) lidera el turismo de montaña fuera de la temporada invernal (Tourespaña, 2009), generando importantes beneficios socioeconómicos (Sánchez-Sanz, 2014), convirtiéndose como alternativa y medida de adaptación al cambio climático para el turismo de esquí. Asimismo, conviene trascender que, el clima es la variable más importante para seleccionar España como destino de montaña para el turismo internacional (Tourespaña, 2009).

En cambio, no se han realizado hasta el momento estudios específicos sobre clima y senderismo. Así, excluyendo al turismo de sol y playa y las aproximaciones generales, sólo se encuentran investigaciones concretas, por ejemplo, para: la práctica del sky (Shih, et al., 2009; Tang y Jang, 2012; Falk, 2015); el golf (Scott y Jones, 2006; Nicholls et al., 2008); el turismo urbano (Scott et al., 2008; Dubois et al., 2009, Matzarakis y Endler, 2009; Rutty y Scott 2010; Scott et al., 2016); o la visita a zoológicos (Aylen y Kevin-Albertson, 2014; Hewer y Gough, 2016). Para el caso del turismo de montaña, y fuera de España, hemos encontrado los trabajos de afiliación general de George (1993), Dubois et al. (2009), Scott et al. (2008), Jänicke, Abegg y Steiger (2015) y Dubois et al. (2016). Únicamente el Ministerio Federal de Medio Ambiente de Canadá en los años 70 del s.XX ha valorado el ambiente atmosférico para la práctica de lo que ellos nombraron caminatas turísticas (ver Gómez-Martín, 2000).

Por todo ello, surge la necesidad de abordar este estudio, como primera aproximación, dedicada a las preferencias climáticas para la práctica del senderismo en España.

\section{MÉTODO}

El presente trabajo tiene por objeto realizar una primera aproximación para el evaluar el grado de aptitud de las condiciones atmosféricas para la práctica turística del senderismo.

El cuerpo metodológico para poder evaluar la calidad/aptitud del tiempo y el clima para la actividad turística (entendiendo el tiempo y el clima como un recurso o un atractivo turístico) ha sido desarrollada por Besancenot (1990) y de Freitas (1990; 2001; 2003 y 2005). Posteriormente, ha sido completada y emparejada por Martínez-Ibarra (2006). Considerando dichas propuestas el mismo queda como aparece en la tabla 1.

Al respecto, cabe señalar que, las facetas estética, física y térmica se refieren a las características del ambiente atmosférico para esas tres componentes. Así, por ejemplo, las cualidades estéticas del estado atmosférico vienen definidas por la insolación, la nubosidad, la duración del día, la visibilidad y la calidad del aire.

Por lo que respecta a la faceta física, debemos considerar que ciertos elementos climáticos se muestran como un agente físico material o con capacidad para movilizar materia. La precipitación (líquida o sólida), el hielo o la existencia de partículas en suspensión (que condicionan la calidad del aire) se muestran como agentes físicos materiales; mientras que el viento, por la segunda de las cuestiones aludidas, cuando su fuerza supera cierto umbral, puede resultar molesto, incluso condicionar la propia seguridad del turista. 
Tabla 1. Matriz tiempo/clima y turismo

\begin{tabular}{|c|c|c|c|c|c|c|c|c|c|c|c|c|c|}
\hline & \multicolumn{12}{|c|}{ FACETAS CLIMÁTICAS } \\
\hline & & \multicolumn{12}{|c|}{ ESTÉTICA $(a, b, c, d, i)$} \\
\hline \multirow{4}{*}{$\begin{array}{l}\text { EXIGENCIAS } \\
\text { FUNDAMENTALES } \\
\text { DEL TURISTA }\end{array}$} & Seguridad & & & & & & & & & & & & \\
\hline & Disfrute & & & & & & & & & & & & \\
\hline & Confort & & & & & & & & & & & & \\
\hline & Salud & & & & & & & & & & & & \\
\hline \multicolumn{2}{|c|}{ Variables Meteorológicas } & $\mathrm{a}$ & $b$ & $\mathrm{C}$ & $\mathrm{d}$ & $\mathrm{e}$ & $f$ & $g$ & $\mathrm{~h}$ & $\mathrm{i}$ & j & $\mathrm{k}$ & 1 \\
\hline & & \multicolumn{12}{|c|}{ FÍSICA $(e, f, g, h, i)$} \\
\hline \multirow{4}{*}{$\begin{array}{l}\text { EXIGENCIAS } \\
\text { FUNDAMENTALES } \\
\text { DEL TURISTA }\end{array}$} & Seguridad & & & & & & & & & & & & \\
\hline & Disfrute & & & & & & & & & & & & \\
\hline & Confort & & & & & & & & & & & & \\
\hline & Salud & & & & & & & & & & & & \\
\hline \multicolumn{2}{|c|}{ Variables Meteorológicas } & $\mathrm{a}$ & $b$ & $\mathrm{C}$ & $\mathrm{d}$ & $\mathrm{e}$ & $f$ & $g$ & $\mathrm{~h}$ & $\mathrm{i}$ & $\mathrm{j}$ & $\mathrm{k}$ & 1 \\
\hline & & \multicolumn{12}{|c|}{ TÉRMICA $(e, j, k, l)$} \\
\hline \multirow{4}{*}{$\begin{array}{l}\text { EXIGENCIAS } \\
\text { FUNDAMENTALES } \\
\text { DEL TURISTA }\end{array}$} & Seguridad & & & & & & & & & & & & \\
\hline & Disfrute & & & & & & & & & & & & \\
\hline & Confort & & & & & & & & & & & & \\
\hline & Salud & & & & & & & & & & & & \\
\hline \multicolumn{2}{|c|}{ Variables Meteorológicas } & a & $b$ & C & $d$ & $\mathrm{e}$ & $f$ & g & $\mathrm{h}$ & $\mathrm{i}$ & j & $\mathrm{k}$ & I \\
\hline
\end{tabular}

Fuente: inspirada en Besancenot, J.P. (1990), de Freitas, C.R. (1990; 2001; 2003; 2005) y modificada a partir de Martínez-Ibarra (2006) y Martínez-lbarra y Gómez-Martín (2013).

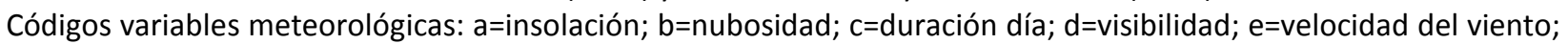
$\mathrm{f}=$ precipitación líquida; $\mathrm{g}=$ precipitación sólida; $\mathrm{h}=$ hielo; $\mathrm{i}$ : calidad del aire; j= radiación solar; $\mathrm{k}=$ temperatura; $\mathrm{I}$ : humedad.

La faceta térmica se relaciona con el confort térmico. Por tanto, dicha característica del estado atmosférico (la térmica), queda marcada por la temperatura, la humedad y el viento (ambos condicionan la sensación térmica), y la radiación solar (variable fundamental en el balance radiativo entre el cuerpo humano y el ambiente que le rodea).

A su vez, los distintos parámetros que conforman las facetas atmosféricas, influyen en la sensación de seguridad, disfrute, confort y la salud de los turistas.

Según Scott et al. (2008), las preferencias del turista en materia climática pueden ser determinadas a partir de tres procedimientos: por un lado, a través de la consulta a expertos o profesionales en turismo; de otro, analizando la relación entre el comportamiento del turista y las condiciones atmosféricas y climáticas; y, por último, a partir de cuestionarios climáticoturísticos realizados a los turistas. En este trabajo se ha optado por la tercera de las estrategias.

Por consiguiente, para llevar a término esta primera aproximación a las preferencias y percepciones sobre las condiciones atmosféricas para la práctica del senderismo en España se ha elaborado una encuesta. Ésta se ha desarrollado "ex situ" (Dubois et al., 2016), a través de internet, como en otros trabajos (p.ej. Dubois et al., 2009; Dubois et al., 2016). 
Los cuestionarios se distribuyeron primero a través de correo electrónico en formato texto, y, posteriormente, por medio de la red social Facebook, haciendo uso de la plataforma gratuita survio (http://www.survio.com/es/). En total se han obtenido 142 cuestionarios útiles, durante el periodo abril-mayo de 2016. En la mayor parte de los casos (casi un 85\%), la experiencia en la práctica del senderismo por parte de los encuestados ha sido igual o mayor a 1 año, con una frecuencia media de salidas igual o superior a una vez al mes (aproximadamente el $77 \%$ de los encuestados), y la mayoría contaban con estudios superiores finalizados (prácticamente un 55\%). Ello, en cierta forma, resuelve los problemas asociados con la cantidad de población estudiada $(n=142)$, en tanto que las respuestas de los individuos pueden considerarse bastante representativas (Tabla 2).

Tabla 2. Ficha técnica de la encuesta

\begin{tabular}{ll} 
Especificación & Características \\
Tipo de encuesta & "Ex-situ" \\
Medio & Internet (correo electrónico y plataforma \\
& survio) \\
Periodo & Abril y mayo de 2016 \\
Características de la muestra & Experiencia en senderismo $\geq 1$ año $(85 \%)$ \\
& Estudios superiores $(\approx 55 \%)$ \\
Cuestionarios válidos & $\mathrm{n}=142$ \\
\hline
\end{tabular}

Elaboración propia.

Las preguntas de la encuesta se confeccionaron teniendo en cuenta la matriz de aptitud climático-turística mostrada en la tabla 1 (relativa a las facetas climáticas y las exigencias fundamentales de los turistas en materia climática). Los trabajos que utilizan la técnica de la encuesta para valorar las preferencias climático-turísticas, también han sido de gran ayuda. Esto es, los de Gómez-Martín, 2006; Morgan et al., 2000; de Freitas et al., 2008; Scott et al., 2008; Dubois et al., 2009; Rutty y Scott, 2010; Moreno, 2010; Gómez-Martín, Armesto-López y Martínez-Ibarra, 2014; Jänicke, Abegg y Steiger, 2015; Rutty y Scott, 2015; y Dubois et al., 2016).

En este caso, se ha prestado atención a las siguientes variables: la nubosidad, la temperatura, la visibilidad, la precipitación y la velocidad del viento. Además, se ha preguntado a los encuestados por la estación del año más adecuada para la práctica del senderismo. Con ello, se han buscado las preferencias y los límites por facetas climáticas (estética, física y térmica) para la práctica del senderismo. En algunos casos, también se ha prestado atención a la importancia concedida a diferentes parámetros climáticos (p.ej. el tipo de precipitación).

\section{RESULTADOS}

En primer lugar, se preguntó sobre las preferencias por el estado del cielo. Concretamente, se les pidió a los encuestados que indicaran el estado del cielo preferido para la práctica del senderismo. La respuesta se planteó de forma cerrada, ofreciéndoles la posibilidad de elegir entre las siguientes posibilidades: Despejado, Poco Nuboso, Nuboso y Muy Nuboso (Tabla 3). 
Tabla 3. Preferencias sobre el estado del cielo para la práctica del senderismo

\begin{tabular}{|ll|}
\hline Estado del cielo & \% de respuestas \\
\hline Despejado & $41,5 \%$ \\
Poco nuboso & $43 \%$ \\
Nuboso & $13,4 \%$ \\
Muy nuboso & $2,1 \%$ \\
\hline
\end{tabular}

Elaboración propia.

Como puede observarse, prácticamente el $85 \%$ de los encuestados prefiere estados de cielo con escasa nubosidad (grados de cobertura nubosa inferior al 30\%). Estos resultados concuerdan con los obtenidos en otros trabajos desarrollados para el turismo el turismo estival en general y de sol y playa en particular, desarrollados en distintos ámbitos (ver Martínez-lbarra y Gómez-Martín, 2013; de Freitas, 2015; Rutty y Scott, 2016; Scott et al., 2016). También comulgan con los obtenidos para el caso concreto del turismo de montaña por Scott et al. (2008). En este sentido, debemos tener presente que la insolación es la segunda variable climática en importancia, tras la precipitación, para la práctica del turismo de montaña en verano en la zona alpina alemana de Baviera (Jänicke, Abegg y Steiger, 2015).

A continuación, con formato de respuesta abierta, se preguntó sobre el rango de temperatura ideal para la práctica del senderismo, así como por los límites (máximos y mínimos) a partir de los cuáles dicha práctica no se desarrollaría (Tabla 4).

Tabla 4. Preferencias y límites térmicos (máximas diarias) para la práctica del senderismo

\begin{tabular}{|llllll|} 
Umbral ideal & Respuestas & $\begin{array}{l}\text { Límite } \\
\text { superior }\end{array}$ & Respuestas & $\begin{array}{l}\text { Límite } \\
\text { inferior }\end{array}$ & Respuestas \\
\hline$<10 \circ \mathrm{C}$ & $2,9 \%$ & $<25 \circ \mathrm{C}$ & $0,7 \%$ & $<-10 \circ \mathrm{C}$ & $8,7 \%$ \\
{$[10 \circ \mathrm{C}, 14 \circ \mathrm{C}]$} & $18 \%$ & {$[25 \circ \mathrm{C}$,} & $21,6 \%$ & {$[-10 \circ \mathrm{C}$,} & $50,7 \%$ \\
& & $29 \circ \mathrm{C}]$ & & $-1 \circ \mathrm{C}]$ & \\
{$[15 \circ \mathrm{C}, 19 \circ \mathrm{C}]$} & $60,4 \%$ & {$[30 \circ \mathrm{C}$,} & $49,6 \%$ & {$[0 \circ \mathrm{C}$,} & $33,3 \%$ \\
& & $34 \circ \mathrm{C}]$ & & $9 \circ \mathrm{C}]$ & \\
$\geq 20 \circ \mathrm{C}$ & $18,7 \%$ & $\geq 35 \circ$ & $28,1 \%$ & $\geq 10 \circ$ & $7,2 \%$ \\
\hline
\end{tabular}

Elaboración propia.

Según los encuestados el rango ideal de temperaturas máximas diarias para practicar el senderismo es de 15 a 19으 (16.9으 en forma de valor medio). Estos resultados se aproximan a los obtenidos para el turismo de montaña por Scott et al. (2008) y Jänicke, Abegg y Steiger (2015), si bien son sensiblemente más bajos. Así, los primeros obtuvieron un valor ideal de 20,5우 mientras que los segundos determinaron el umbral ideal de 21.3 a 24.9 으, y como valor ideal los 23.1 으.

En el caso del límite superior, la horquilla de valores obtenida ha sido de 30-34으, con un valor medio de $31.1^{\circ} \mathrm{C}$. Resultados muy similares a los establecidos para el turismo de montaña por otros autores: 30ㄷ Dubois et al. (2009) y 29.4으 Jänicke, Abegg y Steiger (2015). 
Por su parte, los encuestados han indicado que el límite inferior a partir del cual no realizarían senderismo quedaría comprendido entre los -10 y el $-1 ㅇ$ C, con un valor medio de 3.2ㄷ. En otros trabajos se han propuesto valores bastante distintos: 9o C Dubois et al. (2009) y 14,6으 Jänicke, Abegg y Steiger (2015). Quizás las diferencias se encuentren en la estación del año tomada en consideración por algunos autores, el verano en el caso de Jänicke, Abegg y Steiger (2015). También conviene indicar que se ha trabajado con una muestra que manifiesta tener cierta experiencia en la práctica del senderismo. Ante dichas características de la muestra, es factible considerar su predisposición para disfrutar de los paseos de invierno con ropa adecuada. Al respecto, recuérdense los límites del índice humidex propuestos por el Ministerio Federal de Medio Ambiente de Canadá en los años 70 del s.XX para la práctica del esquí o el uso de la motonieve. Efectivamente, los límites inferiores establecidos por los autores para la práctica de dichas actividades fueron de $-14,4$ y -21,1, respectivamente (ver Gómez-Martín, 2000). Además, debe también traerse a colación los resultados de Steiger et al. (2016). Estos autores indican que los turistas que realizan ocio ligado al desarrollo de actividad física en montaña toleran más las bajas temperaturas.

La visibilidad es otra cualidad del estado atmosférico de primer orden en la práctica del senderismo, ya que en esta modalidad turística, la contemplación y el disfrute del paisaje, es fundamental. Además cobra importancia en la seguridad del turista, ya que un ambiente con niebla puede asociarse a determinados peligros por falta de visibilidad. En materia climáticoturística, sólo ha sido tomada en consideración por la propuesta elaborada por el Ministerio Federal de Medio Ambiente de Canadá. En este caso, la pregunta se ha elaborado con respuesta cerrada, marcando las siguientes posibilidades: ausencia de nieblas y brumas; existencia de bruma o calima (visibilidad entre 1 y $10 \mathrm{~km}$ ); existencia de niebla débil (visibilidad entre 500 y $1.000 \mathrm{~m}$.); niebla moderada (visibilidad entre 50 y $500 \mathrm{~m}$.); y niebla densa (visibilidad inferior a $50 \mathrm{~m})$. Se han definido estos umbrales a partir de Llorente-Martínez (2003).

Tabla 5. Preferencias sobre el nivel de visibilidad para la práctica del senderismo

\begin{tabular}{|ll|}
\hline Nivel de visibilidad & Respuestas \\
\hline Ausencia de nieblas o brumas & $70,7 \%$ \\
Bruma o calima & $18,6 \%$ \\
Niebla débil & $7,9 \%$ \\
Niebla moderada & $2,9 \%$ \\
Niebla densa & $0 \%$ \\
\hline
\end{tabular}

Elaboración propia.

Respecto a la transparencia atmosférica, se prefieren los ambientes con buena visibilidad (Tabla 5), al igual que reflejan los resultados del trabajo desarrollo por el Ministerio Federal de Medio Ambiente de Canadá.

La precipitación (su ausencia o escasa manifestación) es la variable climática más importante para el turista en Francia (Dubois et al., 2009); y también para la práctica del turismo de montaña en la zona alpina alemana de Baviera (Jänicke, Abegg y Steiger, 2015). Para testar las preferencias sobre este parámetro hemos diferenciado entre: intensidad o cantidad de precipitación diaria; el ideal de cantidad de precipitación diaria; el límite de precipitación diaria a partir del cual no se practicaría senderismo; la intensidad de precipitación admisible; y el tipo 
de precipitación más permisiva para la práctica del senderismo (Tabla 6). Algunas de estas cuestiones nunca se habían tratado en climatología turística (concretamente, la intensidad y el tipo de precipitación).

Respecto a la primera pregunta, llama la atención que, los encuestados han otorgado mayor importancia a la intensidad que a la cantidad de la precipitación para el desarrollo de la práctica del senderismo (78,2\% frente al $12,4 \%)$. Como se ha demostrado en otros trabajos de climatología turística, lo ideal para el turista es que no se produzca precipitación $(87,6 \%)$, siendo el umbral máximo diario de precipitación admitido por la mayoría el de los $10 \mathrm{~mm}$. Este valor dobla al límite más permisivo propuesto en climatología turística. Esto es, los $5 \mathrm{~mm}$ definidos por Martínez-Ibarra (2008a) para el turismo de sol y playa. No obstante, cabe apuntar que el segmento aquí analizado puede considerarse más "resistente" a este inconveniente físico. De hecho, para estos dos casos concretos, la actividad desarrollada por el senderista no puede compararse con la vinculada con el disfrute del sol y la playa. Téngase en cuenta, por ejemplo, el poder aislante de las prendas que pueden utilizarse cuando se practica senderismo.

Para el caso de la intensidad de la precipitación se han considerado opciones de respuesta cerrada. Al respecto, los límites establecidos están basados en Cuadrat-Prats y PitaLópez (1997). En este sentido, los encuestados prefieren intensidades débiles de precipitación $(48,9 \%)$ o, a lo sumo, moderadas (44\%).

Por lo que respecta a la cantidad y a la forma (agua, nieve o granizo), la mayoría de los encuestados se decantan por otorgar mayor relevancia a la cantidad que al tipo de precipitación (55,8\% frente al $26,8 \%)$. En cuanto a la forma, los encuestados han manifestado una mayor permisividad por la precipitación líquida. En último lugar ha quedado la sólida en forma de granizo. Por tanto, y en orden de preferencia decreciente, el trinomio Agua-Nieve-Granizo ha sumado el $50 \%$ de las respuestas.

Tabla 6. Preferencias sobre la precipitación para la práctica del senderismo

\begin{tabular}{llllll|}
$\begin{array}{l}\text { Cantidad } \\
\text { versus } \\
\text { intensidad }\end{array}$ & Respuestas & $\begin{array}{l}\text { Ausencia } \\
\text { versus } \\
\text { presencia }\end{array}$ & Respuestas & $\begin{array}{l}\text { Cantidad Respuestas } \\
\text { Máxima }\end{array}$ & $\begin{array}{l}\mathbf{p} \text { diaria } \\
\text { (mm) }\end{array}$ \\
\hline $\begin{array}{l}\text { Cantidad } \\
\text { Intensidad }\end{array}$ & $12,7 \%$ & Ausencia & $87,6 \%$ & $\leq 10$ & $44,37 \%$ \\
\hline
\end{tabular}

Elaboración propia.

Respecto a la velocidad del viento, conviene tener presente que la pregunta se ha presentado en formato cerrado, tomando como referencia la escala de Beaufourt, como en el trabajo de Gómez-Martín (2004). Las preferencias por los turistas para la práctica del senderismo son las brisas muy débiles ( 6 a $11 \mathrm{Km} / \mathrm{h}$ ) con el $21 \%$ de los casos; las calmas ( 0 a 1 $\mathrm{Km} / \mathrm{h}$ ) y las brisas débiles (12 a $19 \mathrm{Km} / \mathrm{h}$ ), con el $18,1 \%$ de los casos, en ambas situaciones; y las brisas moderadas (20 a $28 \mathrm{Km} / \mathrm{h}$ ), con el 15,2\%. Estos resultados son similares a los obtenidos en otros segmentos del turismo y en otras áreas geográficas (ver Martínez-lbarra y GómezMartín, 2013; Scott et al., 2016). En cuanto al límite superior de velocidad del viento, a partir del 
cual no se desarrollaría la práctica del senderismo, el más indicado ha sido el de $50 \mathrm{Km} / \mathrm{h}$. Un umbral próximo al estimado como inaceptable para la práctica turística por Rutty y Scott (2010), Wirth (2014) y Guo (2015).

Por último, se ha pedido a los encuestados que ordenen según sus preferencias las estaciones del año para el desarrollo de la práctica turística del senderismo. En este caso los resultados obtenidos han sido: primavera $(55,6 \%)$, otoño $(26,1 \%)$, invierno $(11,3 \%)$ y verano $(4,2 \%)$. La opción más votada es la seleccionada por el turismo internacional como más interesante para la práctica del turismo de montaña en España (Tourespaña, 2009).

\section{CONCLUSIONES}

Se ha presentado una primera aproximación sobre las preferencias climáticas para la práctica del senderismo en España. A pesar de la necesidad de ahondar más en la cuestión a partir del análisis de muestras más numerosas, se pueden extraer una serie de conclusiones de importancia, dada la relevancia de la muestra analizada, por su experiencia en la actividad que se valora y su nivel formativo.

Tabla 7. Condiciones climáticas óptimas para el desarrollo de diversas prácticas turísticas

\begin{tabular}{|c|c|c|c|c|c|c|}
\hline \multirow[t]{2}{*}{ Región analizada } & \multirow[t]{2}{*}{$\begin{array}{l}\text { Segmento } \\
\text { turístico }\end{array}$} & \multicolumn{4}{|c|}{$\begin{array}{l}\text { Condiciones climáticas óptimas } \\
\text { (escala temporal diaria) }\end{array}$} & \multirow[t]{2}{*}{ Fuente } \\
\hline & & $\begin{array}{l}\text { Temperatura } \\
(\stackrel{\circ}{ })\end{array}$ & $\begin{array}{l}\text { Precipitación } \\
(\mathrm{mm})\end{array}$ & $\begin{array}{l}\text { Nubosidad } \\
\text { (\%) }\end{array}$ & $\begin{array}{l}\text { Viento } \\
(\mathrm{Km} / \mathrm{h})\end{array}$ & \\
\hline Francia & $\begin{array}{l}\text { General } \\
\text { (verano) }\end{array}$ & {$[25,33]$} & 0 & $\leq 25$ & $<28,8$ & $\begin{array}{l}\text { Besancenot } \\
\text { et al. (1978) }\end{array}$ \\
\hline Brasil & General & $>28$ & $\leq 1$ & $\leq 40$ & $<10,8$ & $\begin{array}{l}\text { Barbière } \\
\text { (1981) }\end{array}$ \\
\hline $\begin{array}{c}\text { Global } \\
\text { (Climas } \\
\text { templados) }\end{array}$ & $\begin{array}{l}\text { General } \\
\text { (verano) }\end{array}$ & {$[25,31]$} & 0 & $\leq 25$ & $<28,8$ & $\begin{array}{l}\text { Besancenot } \\
\text { (1990) }\end{array}$ \\
\hline $\begin{array}{l}\text { Cataluña } \\
\text { (España) }\end{array}$ & $\begin{array}{l}\text { General } \\
\text { (verano) }\end{array}$ & {$[22,28]$} & 0 & $\leq 25$ & $<28,8$ & $\begin{array}{c}\text { Gómez-Martín } \\
\text { (2000) }\end{array}$ \\
\hline $\begin{array}{l}\text { Jardines del Rey } \\
\text { (Cuba) }\end{array}$ & General & {$[22,31]$} & 0 & $\leq 38$ & $<28,8$ & $\begin{array}{c}\text { Batista y } \\
\text { Matos (2004) }\end{array}$ \\
\hline $\begin{array}{c}\text { Provincia de } \\
\text { Alicante (España) }\end{array}$ & Sol y Playa & {$[28,31]$} & 0 & $\begin{array}{l}\quad \geq 75 \\
\text { (insolación) }\end{array}$ & $<30,4$ & $\begin{array}{c}\text { Martínez- } \\
\text { Ibarra (2006, } \\
\text { 2008a, 2008b) }\end{array}$ \\
\hline $\begin{array}{l}\text { Canadá, Nueva } \\
\text { Zelanda y Suecia }\end{array}$ & Sol y Playa & 26,8 & - & $\leq 25$ & {$[1,9]$} & $\begin{array}{l}\text { Scott et al. } \\
\text { (2008) }\end{array}$ \\
\hline China & Urbano & 20 & $<15$ (minutos) & $\leq 25$ & {$[1,9]$} & Guo (2015) \\
\hline $\begin{array}{l}\text { Canadá, Nueva } \\
\text { Zelanda y Suecia }\end{array}$ & Montaña & 20,5 & - & $\leq 25$ & - & $\begin{array}{l}\text { Scott et al. } \\
(2008)\end{array}$ \\
\hline $\begin{array}{c}\text { Alpes } \\
\text { (Alemania) }\end{array}$ & Montaña & {$[21,25]$} & & $\begin{array}{l}\text { "ligeramente } \\
\text { nuboso" }\end{array}$ & & $\begin{array}{l}\text { Steiger et al. } \\
\quad(2016)\end{array}$ \\
\hline España & Senderismo & {$[15 \circ \mathrm{C}, 19 \circ \mathrm{C}]$} & 0 & $\leq 30$ & $<28$ & Este estudio \\
\hline
\end{tabular}

Elaboración propia.

En primer lugar, cabe subrayar las preferencias por los estados del cielo poco nubosos o despejados para la práctica del senderismo; al igual que sucede en otras actividades turísticas (Tabla 7). En este sentido, debe tenerse en cuenta que esta faceta estética ejerce una influencia 
importante en las sensaciones de disfrute, el confort y la salud. Ciertamente, se ha demostrado que la insolación explica por si sola entre el 15 y el $40 \%$ de la sensación de disfrute en el ser humano (Winslow y Herrington, 1935; Auliciems, 1976 y 1978).

En cuanto a la faceta térmica, la práctica del senderismo parece mostrarse mucho más exigente que otras opciones de ocio y recreación en confort térmico durante el periodo cálido, especialmente con respecto al turismo de sol y playa (Tabla 7). Sin embargo, parece mucho más flexible para soportar temperaturas bajas. Ello puede deberse a las tasas de producción de calor metabólico (ver Martínez-Ibarra, 2006) y a las opciones de adaptación a las bajas temperaturas a través del uso de la vestimenta, alternativa que no existe para los ambientes cálidos.

De otro lado, como se ha indicado, en el desarrollo de la actividad del senderismo juega un papel importante el disfrute a través de la contemplación del paisaje, de ahí las exigencias pronunciadas por los encuestados en relación con la faceta estética visibilidad.

Al igual que en otros segmentos de demanda turística, los practicantes del senderismo prefieren que la precipitación (faceta física) no se manifieste cuando desarrollan esta actividad (Tabla 7), ya que puede determinar por si sola la impresión de que el tiempo ha sido totalmente desfavorable (Campbell y Beets, 1977; Cunningham, 1979; Besancenot, 1990). No obstante, también han manifestado mayor flexibilidad ante la presencia de este hidrometeoro. Bien es cierto que se prefiere en su forma líquida y con intensidades débiles, a lo sumo moderadas.

Otra de las facetas físicas del medio atmosférico es el viento. Su presencia puede condicionar, incluso impedir, la sensación de disfrute y, en ocasiones, comprometer la seguridad del turista. Por ello, los encuestados han manifestado la preferencia por ambientes poco ventosos, como en el resto de las modalidades turísticas (Tabla 7). Con todo, han declarado ser más tolerantes que otras tipologías de turistas en cuanto a los límites superiores.

Estas particularidades sobre las valoraciones climáticas para la práctica del senderismo se han proyectado también en la preferencia estacional para desarrollar esta actividad de ocio. En este caso el verano es la estación menos deseada. De hecho, para practicar senderismo los encuestados han manifestado su predilección por el otoño y, especialmente, la primavera. Por tanto, esta práctica, tan importante en el turismo de montaña, se puede convertir en su principal aliada para adaptarse al cambio global y superar la estacionalidad turística.

Para futuros trabajos se plantea seguir ahondando en el conocimiento de las relaciones clima-senderismo, sobre la base de la práctica inexistencia de investigaciones a este respecto, y la importancia de esta práctica en la actividad turística.

\section{REFERENCIAS BIBLIOGRÁFICAS}

Auliciems, A. (1976). Weather perception: a subtropical winter study. Weather, 9, 312-316. doi: 10.1002/j.1477-8696.1976.tb04467.x

Auliciems, A. (1978). Mood dependency on low intensity atmospheric variability. International Journal of Biometeorology, 1, 20-32. doi: 10.1007/BF01553137

Aylen, J., y Kevin-Albertson, G. C. (2014). The impact of weather and climate on tourist demand: the case of Chester Zoo. Climatic Change, 2,183-197. doi:10.1007/s10584-014-1261-6 
Barbière, E.B. (1981): O fator climatico nos sistemas territoriais de recreação. Revista Brasileira de Geografia, 2, 145-265.

Batista Tamayo, L. M. y Matos Pupo, F. (2004). La aptitud climática del destino turístico Jardines del Rey (Cuba). Los tipos de tiempo. En J. C. García Codrón, Liaño, C. D., P. F. de Arróyabe Hernáez, C. Garmendia Pedraza, D. Rasilla Álvarez (Eds.), El clima entre el mar y la montaña (561-570). Santander: Publicaciones de la Asociación Española de Climatología.

Besancenot, J. P. (1990). Clima et tourismes. París: Massom.

Besancenot, J.P., Mounier, J. y de Lavenne, F. (1978). Les conditions climatiques du tourisme littotal: une méthode de recherche comprénshive. Norois, 99, 357-382.

Campbell, D.E., y Beets, J.L. (1977). Meteorological variables and behavior: An annotated bibliography. JSAS Catalog of Selected Documents in Psychology, 7, 1.

Cunningham, M.R. (1979). Weather, mood and helping behavior: Quasi Experiments with the Sunshine Samaritan. Journal of Personality and Social Psychology, 11, 1947-1956. doi: 10.1037/0022-3514.37.11.1947

Dubois, G., Ceron, J.P., van de Walle, I., y Picard, R. (2009). CREDOC. Climat, meteorology et frequentation touristique, rapport final. París: Ministere de l'Ecologie, de l'Energie, du Développement durable et de la Mer.

Dubois, G., Ceron, J.P., Gössling, S., y Hall, C.M. (2016). Weather preferences of French tourists: for climate change impact assessment. Climatic Change, 136, 339-351. doi:10.1007/s10584016-1620-6

de Freitas, C. R. (1990). Recreation climate assessment. International Journal of Climatology, 1, 89-103. doi:10.1002/joc.3370100110

de Freitas, C. R. (2001). Theory, concepts and methods in tourism climate research. En A. Matzarakis, y C. R. de Freitas (Eds.), Proceedings of the First International Workshop on Climate, Tourism and Recreation (pp. 3-20). Porto Carras: International Society of Biometeorology, Commission on Climate Tourism and Recreation.

de Freitas, C. R. (2003). Tourism climatology: evaluating environmental information for decisionmaking and business planning in the recreation and tourism sector. International Journal of Biometeorology, 1, 45-54. doi:10.1007/s00484-003-0177-z

de Freitas, C. R. (2005). The climate-tourism relationship and its relevance to climate change impact assessment. En C. M. Hall, y J. Higham (Eds.), Tourism, recreation and climate change (pp. 29-43). Toronto: Channel view publications.

de Freitas, C.R. (2015). Weather and place-based human behavior: recreational preferences and sensitivity. International Journal of Biometeorology, 59, 55-63. doi:10.1007/s00484-0140824-6

de Freitas C.R., Scott D., y McBoyle, G. (2008). A second generation climate index for tourism (CIT): specification and verification. International Journal Biometeorology, 52(5), 399-407. doi:10.1007/s00484-014-0824-6

Falk, M. (2015). The Demand for Winter Sports: Empirical Evidence for the Largest French SkiLift Operator. Tourism Economics, 3, 561-580. doi:10.5367/te.2013.0366

George, D. (1993). Weather and mountain activities. Weather, 48, 404-410. doi:10.1002/j.14778696.1993.tb05829.x

Gómez-Martín, Mạ.B. (2000). Clima y turismo en Cataluña: Evaluación del potencial climáticoturístico de la estación estival (Tesis doctoral). Universidad de Barcelona.

Investigaciones Turísticas

$N^{\circ} 13$, enero-junio 2017, pp. 164-177 
Gómez-Martín, Mạ.B. (2004). Percepción de la demanda y métodos de evaluación de la potencialidad turística de los recursos atmosféricos en Cataluña. Documents d'Anàlisi Geogràfica Regional, 44, 43-70.

Gómez-Martín, Mà.B. (2005). Weather, climate and tourism a geographical perspective. Annals of Tourism Research, 3, 571-591. doi:10.1016/j.annals.2004.08.004

Gómez-Martin, Mà. B. (2006). Climate potential and tourist demand in Catalonia (Spain) during the summer season. Climate Research, 32, 75-87. doi:10.3354/cr032075

Gómez-Martín, Mạ. B., Armesto-López, X.A., y Martínez-Ibarra, E. (2014). The Spanish tourist sector facing extreme climate events: a case study of domestic tourism in the heat wave of 2003. International Journal of Biometeorology, 58, 781-797. doi: 10.1007/s00484-013-06596

Gössling, S., Bredberg, M., Randow, A., Svensson, P., y Swedlin, E. (2006). Tourist Perceptions of Climate Change: A study of international tourists in Zanzibar. Current Issues in Tourism, 4-5, 419-435.

Guo, P. (2015). Weather and air quality preferences of urban tourists in China (Tesis Doctoral). Universidad de Waterloo.

Hamilton, J. M., y Lau, M. (2005). The role of climate information in tourist destination choice decision making. En Gössling, S., y Hall, C. M. (Eds.), Tourism and global environmental change (229-250). London: Routledge.

Hewer, M.J., y Gough, W.A. (2016). Weather sensitivity for zoo visitation in Toronto, Canada: a quantitative analysis of historical data. International Journal of Biometeorology, 60, 16451660. doi:10.1007/s00484-016-1154-7

Jänicke, L., B. Abegg and R. Steiger 2015. Wheather preferences of summer tourists in mountain environments: a case study in the Bavarian Alps, Germany. En O.C. Demiroğlu., C.R. de Freitas., D. Scott., M.L. Kurnaz., y D. Ünalan (Eds.), Proceedings of the 4th International Conference on Climate, Tourism and Recreation (CCTR) 2015 (pp. 63-67). Istambul: The Commission on Climate, Tourism and Recreation (CCTR) of the International Society of Biometeorology (ISB).

Mintel International Group (1991). Special report-Holidays. Leisure Intelligence. London: Mintel International Group.

Morgan, R., Gatell, E., Junyent, R., Micallef, A., Özhan, E., y Williams A. T. (2000). An improved user-based beach climate index. Journal Coastal Conservation, 6(1), 41-50.

Kozak, M. (2002). Comparative analysis of tourist motivations by nationality and destinations. Tourism Management, 3, 221-232.

Llorente-Martínez, F. (2003). Meteorología (VII): la visibilidad y los factores meteorológicos que influyen en ella. RAM.

Lohmann, M., y Kaim, E. (1999). Weather and holiday destination preferences image, attitude and experience. Tourism Review, 2, 54-64. doi:http://dx.doi.org/10.1108/eb058303

Martínez-Ibarra, E. (2006). Consideraciones geográficas en torno al binomio clima-turismo: aplicación al litoral alicantino (Tesis doctoral). Universidad de Alicante. 
Martínez-Ibarra, E. (2008a). Tipos de tiempo para el turismo de sol y playa en el litoral alicantino. Estudios Geográficos, 135-155. doi:http://dx.doi.org/10.3989/egeogr.2008.i264.82

Martínez-Ibarra, E. (2008b). Evaluación de la aptitud climático-turística para el turismo de sol y playa en Alicante (1974-2003). Investigaciones Geográficas, 45, 141-162.

Martínez-Ibarra, E. (2016). Climate, water and tourism: causes and effects of droughts associated with urban development and tourism in Benidorm (Spain). International Journal of Biometeorology, 5, 487-501. doi:10.1007/s00484-014-0851-3

Martínez-Ibarra, E., y Gómez-Martín, Mä.B. (2013). Progress in tourism climatology. En T. Mihalic., y W. C. Gartner (Eds.), Tourism and developments. Issues and challenges (pp. 121137). Toronto: Channel View Publications.

Matzarakis, A., y Endler, C. (2009). Climate and tourism: Urban tourism potential in Freiburg, Germany. Recuperado de http://www.urbanclimate.net/matzarakis/papers/ICUC7_tourism_374543-3090330190123-002.pdf.

Moreno, A. (2010). Mediteranean tourism and climate (change): a survey based study. Tourism and Hospitality, 3, 253-265. doi: http://dx.doi.org/10.1080/1479053X.2010.502384

Nicholls, S. y Holecek, D. F. (2008). Engaging Tourism Stakeholders in the Development of Climate Change Decision-Support Tools: A Case Study from Michigan, USA. Tourism Review International, 1, 25-42. doi: 10.3727/154427208785899966

Nicholls, S., Holecek, D. F., y Noh, J. (2008). Impact of weather variability on golfing activity and implications of climate change. Tourism Analysis, 2, 117-130. doi: $10.3727 / 108354208785664256$

Perry, A. H. (2004). Sports tourism and climate variability. En A. Matzarakis., C.R. de Freitas., y D. Scott (Eds.), Advances in Tourism Climatology (174-179). Universidad de Friburgo.

Rutty, M., y Scott, D. (2010). Will the Mediterranean become 'too hot' for tourism? A reassessment. Tourism, Hospitality Planning and Development, 7, 267-281. doi: http://dx.doi.org/10.1080/1479053X.2010.502386

Rutty, M., y Scott, D. (2015). Bioclimatic comfort and the thermal perceptions and preferences of beach tourists. International Journal of Biometeorology, 37, 37-45. doi:10.1007/s00484014-0820-x

Rutty, M., y Scott, D. (2016). Comparison of Climate Preferences for Domestic and and International Beach Holidays: A Case Study of Canadian Travelers. Atmosphere, 7, 30. doi:10.3390/atmos7020030

Sánchez-Sanz, V. (2014). El fenómeno senderista en España. Análisis por un panel de expertos (Tesis Doctoral). Universidad de Valencia.

Scott, D., Gössling, S., y de Freitas, C.R. (2008). Preferred climates for tourism: Cases studies from Canada, New Zealand and Sweden. Climatic Research, 1, 61-73. doi: https://doi.org/10.3354/cr00774

Scott, D., Jones, B. (2006). The Impact of Climate Change on Golf Participation in the Greater Toronto Area (GTA): A Case Study. Journal of Leisure Research, 3. Recuperado de https://js.sagamorepub.com/jlr/article/view/511.

Scott, D., Jones, B., y Konopek, J. (2008). Implications of climate and environmental change for nature-based tourism in the Canadian Rocky Mountains: A case study of Waterton Lakes

Investigaciones Turísticas

$N^{\circ} 13$, enero-junio 2017, pp. 164-177 
National Park Implications of climate and environmental change for nature-based tourism in the Canadian Rocky Mountains: A case study of Waterton Lakes National Park. Tourism Management, 28, 570-579. doi:http://dx.doi.org/10.1016/j.tourman.2006.04.020

Scott, D., Rutty, M., Amelung, B., y Tang, M. (2016). An inter-comparison of the Holiday Climate Index $(\mathrm{HCl})$ and the Tourism Climate Index $(\mathrm{TCl})$ in Europe. Atmosphere, 80, 1-17. doi:10.3390/atmos7060080

Shih, C., Nicholls, S., Holecek, D.F. (2009). Impact of weather on downhill ski lift ticket sales. Journal of Travel Research, 3, 359-372. doi: 10.1177/0047287508321207

Smith, K. (1993). The Influence of weather and climate on recreation and tourism. Weather, 48, 398-404. doi: 10.1002/j.1477-8696.1993.tb05828.x

Steiger, R., Abegg, B., Jänicke, L. (2016). Rain, rain, go away, come again another day. Weather preferences of summer tourists in mountain enviroments. Atmosphere, 7, 63-74. doi:10.3390/atmos7050063

Tang, C.H., y Hang, S.C. (2012). Hedging weather risk in nature-based tourism business: An example of ski resorts. Journal of Hospitality \& Tourism Research, 2, 143-163. doi: $10.1177 / 1096348010388655$

Tourespaña (2009). Turismo de montaña. Madrid: Instituto de Turismo de España.

UNWTO (2016). UNWTO annual report 2015. Madrid: UNWTO.

Winslow, C.E.A., y Herrington, L.P. (1935). Subjective reactions of human beings to certain outdoor atmospheric conditions. Heating, Piping and air Conditioning Journal, 7, 551-556.

Wirth, K. (2014). Auswirkungen des Klimawandels auf den Tourismus in Mittelmeerraum Prognosen Anhand Einer Umfrag in Munchen (Tesis Doctoral). Universidad de Múnich.

Para citar este artículo: Martínez Ibarra, E.; Pardo Martínez, R. (2017). Preferencias climáticas para la práctica del senderismo en España: resultados preliminares. Investigaciones Turísticas, (13), $164-177$. http://dx.doi.org/10.461/INTURI2017.13.08 